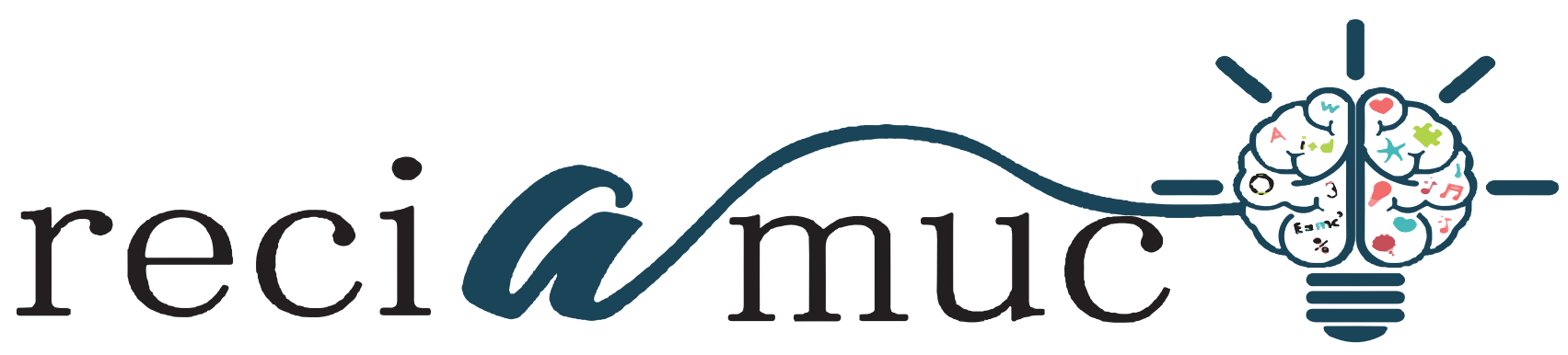

DOI: 10.26820/reciamuc/4.(3).julio.2020.118-126

URL: https://reciamuc.com/index.php/RECIAMUC/article/view/505

EDITORIAL: Saberes del Conocimiento

REVISTA: RECIAMUC

ISSN: 2588-0748

TIPO DE INVESTIGACIÓN: Artículo de Revisión

CÓDIGO UNESCO: 3205 Medicina Interna; 3205.02 Endocrinología

PAGINAS: $118-126$

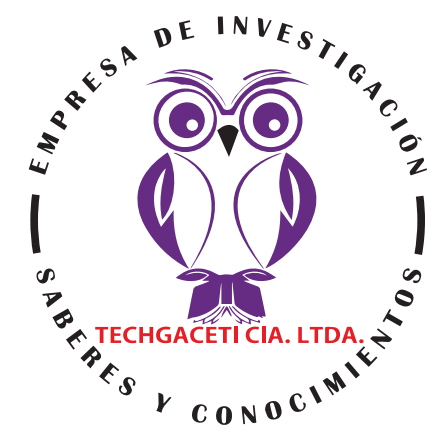

\title{
Utilidad de hemoglobina glicosilada en diabetes tipo 2
} Utility of glycosylated hemoglobin in type 2 diabetes Utilidade da hemoglobina glicosilada no diabetes tipo 2

\section{Katherine Andrea Flores Poveda'; Karen Johanna Quiñonez García2; Denisse Lissette Flores Subía3; Carlos Anthony Cárdenas Choez ${ }^{4}$}

RECIBIDO: 20/05/2020 ACEPTADO: 19/07/2020 PUBLICADO: 30/07/2020

1. Médico; Hospital General Monte Sinai; Guayaquil, Ecuador; katitaflores_p@hotmail.com; (DD https://orcid.org/0000-00031522-7251

2. Master Universitario en Urgencias Emergencias y Catástrofes; Médico; Hospital General Monte Sinai; Guayaquil, Ecuador; nerek003@gmail.com; iD https://orcid.org/0000-0002-9728-9642

3. Médico; Hospital General Monte Sinai; Guayaquil, Ecuador; denisse64@hotmail.com; (D) https://orcid.org/0000-0001-7826$192 X$

4. Médico; Hospital General Monte Sinai; Guayaquil, Ecuador; carlos-baterbass_91@hotmail.com; (iD https://orcid.org/00000002-1386-7741

CORRESPONDENCIA

Katherine Andrea Flores Poveda

katitaflores_p@hotmail.com

Guayaquil, Ecuador

( ) RECIAMUC; Editorial Saberes del Conocimiento, 2020 


\section{RESUMEN}

La diabetes es uno de los principales problemas de salud mundial en todo el mundo. Existen tres tipos de diabetes principalmente: la diabetes tipo 1, diabetes tipo 2 y diabetes gestacional. Entre los tipos de diabetes, la diabetes tipo 2 es la más común, y representa aproximadamente del $85 \%$ a $90 \%$ de todos los casos. Igualmente es la más peligrosa pues su falta de signos y síntomas hacen que su diagnóstico se realice, por lo general, cuando han aparecido alguna de sus complicaciones. Una de las principales pruebas que se usan para medir el nivel promedio de glucosa o azúcar en la sangre es la Hemoglobina Glicosilada (HbA1c). El objetivo fundamental del presente estudio es plasmar lo relativo a los usos de la $\mathrm{HbA1c}$ en pacientes con diabetes tipo 2. El modelo de investigación fue de tipo documental o bibliográfico. Dentro de los resultados de la utilidad de la HbA1c se encontró que es usada como prueba diagnóstica para los estados de disglucemia y diabetes, en combinación con otras pruebas de diabetes como la glucemia en ayunas y la prueba de tolerancia a la glucosa (PTG). Asimismo, en el control del diabético tipo 2 es la principal herramienta que permite el adecuado manejo y ajuste de tratamiento. Por último, resulta un predictor importante de complicaciones asociadas a la diabetes tipo 2 . En conclusión, la $\mathrm{HbA} 1 \mathrm{c}$ constituye en la actualidad la prueba de elección para el seguimiento y el tratamiento crónico de la diabetes, especialmente de la diabetes tipo 2, no obstante, desde el punto de vista diagnóstico sigue siendo un tanto controvertido. La versatilidad de sus usos la convierten en una gran herramienta en la prevención, diagnóstico, tratamiento, control y pronósticos de complicaciones del paciente con diabetes tipo 2.

Palabras clave: Utilidad, Hemoglobina, Glicosilada, Diabetes, Tipo 2.

\section{ABSTRACT}

Diabetes is one of the major global health problems around the world. There are three main types of diabetes: type 1 diabetes, type 2 diabetes and gestational diabetes. Among the types of diabetes, type 2 diabetes is the most common, accounting for about $85 \%$ to $90 \%$ of all cases. It is also the most dangerous because its lack of signs and symptoms make its diagnosis to be made, generally, when any of its complications have appeared. One of the main tests used to measure the average level of glucose or sugar in the blood is Glycosylated Hemoglobin (HbA1c). The main objective of this study is to capture what is related to the uses of $\mathrm{HbA1c}$ in patients with type 2 diabetes. The research model was documentary or bibliographic. Among the results of the usefulness of $\mathrm{HbA1c}$, it was found that it is used as a diagnostic test for the states of dysglycemia and diabetes, in combination with other diabetes tests such as fasting blood glucose and the glucose tolerance test (PTG). Likewise, in the control of type 2 diabetics, it is the main tool that allows adequate management and adjustment of treatment. Lastly, it is an important predictor of complications associated with type 2 diabetes. In conclusion, $\mathrm{HbA} 1 \mathrm{c}$ is currently the test of choice for the follow-up and chronic treatment of diabetes, especially type 2 diabetes, from a diagnostic point of view, it remains somewhat controversial. The versatility of its uses, its use as a great tool in the prevention, diagnosis, treatment, control and prognosis of complications in patients with type 2 diabetes.

Keywords: Utility, Hemoglobin, Glycosylated, Diabetes, Type 2.

\section{RESUMO}

O diabetes é um dos maiores problemas globais de saúde em todo o mundo. Existem três tipos principais de diabetes: diabetes tipo 1, diabetes tipo 2 e diabetes gestacional. Entre os tipos de diabetes, o diabetes tipo 2 é o mais comum, representando cerca de $85 \%$ a $90 \%$ de todos os casos. É também a mais perigosa porque sua falta de sinais e sintomas faz com que seu diagnóstico seja feito, geralmente, quando alguma de suas complicações aparecer. Um dos principais testes usados para medir o nível médio de glicose ou açúcar no sangue é a hemoglobina glicosilada (HbA1c). O objetivo principal deste estudo é capturar o que está relacionado ao uso de HbA1c em pacientes com diabetes tipo 2. O modelo de pesquisa foi documental ou bibliográfico. Entre os resultados da utilidade do HbA1c, verificou-se que ele é utilizado como teste diagnóstico para os estados de disglicemia e diabetes, em combinação com outros testes de diabetes, como glicemia de jejum e teste de tolerância à glicose (PTG). Da mesma forma, no controle de diabéticos tipo 2, é a principal ferramenta que permite o manejo adequado e a adequação do tratamento. Por último, é um importante preditor de complicações associadas ao diabetes tipo 2. Em conclusão, o HbA1c é atualmente o teste de escolha para o acompanhamento e tratamento crônico do diabetes, especialmente do diabetes tipo 2, do ponto de vista diagnóstico, mas permanece um tanto controverso. A versatilidade de seus usos, seu uso como grande ferramenta na prevenção, diagnóstico, tratamento, controle e prognóstico de complicações em pacientes com diabetes tipo 2.

Palavras-chave: Utilidade, hemoglobina glicosilada, diabetes, tipo 2. 


\section{Introducción}

La diabetes es un problema de salud pública importante y una de las cuatro enfermedades no transmisibles (ENT) elegidas por los dirigentes de todo el mundo para intervenir con carácter prioritario. En las últimas décadas se han incrementado continuamente el número de casos y la prevalencia de esta enfermedad. La diabetes es una "grave enfermedad crónica que se desencadena cuando el páncreas no produce suficiente insulina (una hormona que regula el nivel de azúcar, o glucosa, en la sangre), o cuando el organismo no puede utilizar con eficacia la insulina que produce". (Organización Mundial de la Salud - OMS, 2016)

El número de personas con diabetes aumentó de 108 millones en 1980 a 422 millones en 2014. La prevalencia mundial de la diabetes en adultos (mayores de 18 años) ha aumentado del $4,7 \%$ en 1980 al $8,5 \%$ en 2014. Entre 2000 y 2016, se ha registrado un incremento del $5 \%$ en la mortalidad prematura por diabetes. La prevalencia de la diabetes ha aumentado con mayor rapidez en los países de ingresos bajos y medianos que en los países de ingresos altos. La diabetes es una importante causa de ceguera, insuficiencia renal, infarto de miocardio, accidente cerebrovascular y amputación de los miembros inferiores. Se estima que en 2016 la diabetes fue la causa directa de 1,6 millones de muertes. Otros 2,2 millones de muertes eran atribuibles a la hiperglucemia en 2012. Casi la mitad de todas las muertes atribuibles a la hiperglucemia tienen lugar antes de los 70 años de edad. La OMS estima que la diabetes fue la séptima causa principal de mortalidad en 2016. (OMS, 2020)

Existen tres tipos principales de diabetes: la diabetes tipo 1, la diabetes tipo 2 y la diabetes gestacional. La diabetes de tipo 2 (también llamada no insulinodependiente o de inicio en la edad adulta) se debe a una utilización ineficaz de la insulina. Este tipo de diabetes representa la mayoría de todos los casos en todo el mundo. Los síntomas pueden ser similares a los de la diabetes de tipo 1, sin embargo, suelen tener menor intensidad. En virtud de lo cual, la enfermedad puede diagnosticarse cuando ya está en evolución, incluso cuando tiene varios años y en este punto, han aparecido complicaciones. (Organización Panamericana de la Salud - OPS, 2017)

Entre los tipos de diabetes, la diabetes tipo 2 es la más común, y representa aproximadamente del $85 \%$ a $90 \%$ de todos los casos. Se relaciona con factores de riesgo modificables como la obesidad o el sobrepeso, la inactividad física, y las dietas con alto contenido calórico de bajo valor nutricional. (OPS, 2012)

"Aproximadamente 62 millones de personas tienen Diabetes tipo 2 en las Américas. Muchas personas con diabetes tipo 2 ni siquiera saben que la tienen". (OPS, 2019)

Es importante destacar que, dado la facilidad de ignorar la diabetes de tipo 2, especialmente en las etapas iniciales, cuando el paciente no presenta signos, resulta fundamental aplicar estrategias para diagnosticarla de manera temprana y evitar sus complicaciones. Esta enfermedad, una vez presente, afecta muchos de los órganos principales, tales como: el corazón, los vasos sanguíneos, los nervios, los ojos y los riñones. Las complicaciones a largo plazo de esta enfermedad pueden desarrollarse de forma gradual, no obstante, en una última instancia podrían conducir a la pérdida de capacidades o, en el peor de los casos, a la muerte. Las siguientes son algunas de las complicaciones potenciales de la diabetes:

- Enfermedades del corazón y circulatorias. La diabetes aumenta en gran medida el riesgo de enfermedad cardíaca, accidente cerebrovascular, presión arterial alta y estrechamiento de los vasos sanguíneos (aterosclerosis).

- Lesión a los nervios (neuropatía). El exceso de azúcar puede provocar hormi- 
gueo, entumecimiento, ardor o dolor, que generalmente comienza en las puntas de los dedos de los pies o las manos y se extiende gradualmente hacia arriba. Con el tiempo, puedes perder la sensibilidad en las extremidades afectadas.

- El daño en los nervios que controlan la digestión puede causar problemas con náuseas, vómitos, diarrea o estreñimiento. En los hombres, la disfunción eréctil puede ser un problema.

- Daño renal. La diabetes a veces puede causar insuficiencia renal o una enfermedad renal en etapa terminal irreversible, que puede requerir diálisis o un trasplante de riñón.

- Daño en los ojos. La diabetes aumenta el riesgo de enfermedades oculares graves, tales como cataratas y glaucoma, y puede dañar los vasos sanguíneos de la retina y posiblemente ocasionar ceguera.

- Cicatrización lenta. Si no se tratan, los cortes y las ampollas pueden dar lugar a graves infecciones, que tal vez no cicatricen como corresponde. El daño grave podría requerir la amputación del dedo del pie, del pie o de la pierna.

- Deterioro de la audición. Los problemas de audición son más frecuentes en las personas que padecen diabetes.

- Enfermedades de la piel. La diabetes puede hacer que seas más propenso a tener problemas de la piel, como infecciones bacterianas y de hongos.

- Apnea del sueño. La apnea obstructiva del sueño es común en pacientes con diabetes de tipo 2. La obesidad puede ser el principal factor contribuyente a ambas enfermedades. Tratar la apnea del sueño puede disminuir tu presión arterial y hacer que te sientas más descansado, pero no está claro si ayuda a mejorar el control del azúcar en la sangre.
- Enfermedad de Alzheimer. La diabetes de tipo 2 parece aumentar el riesgo de padecer la enfermedad de Alzheimer, aunque no se sabe bien por qué. Cuanto más deficiente sea el control del azúcar en la sangre, mayor será el riesgo. (Mayo Clinic, 2019)

En virtud de lo anterior, es que resulta fundamental un diagnóstico oportuno y temprano, así como el control adecuado de la enfermedad una vez diagnosticada. La HbA1c es una prueba recomendada en todo el mundo como un estándar de atención (SOC por sus siglas en inglés) para evaluar y controlar la diabetes, específicamente la diabetes tipo 2. "El análisis de la hemoglobina glucosilada ( $\mathrm{HbA1c}$ ) en sangre proporciona evidencia sobre los niveles promedio de glucosa en sangre de un individuo durante los dos o tres meses anteriores, que es la vida media predicha de los glóbulos rojos (GR)". (Sherwani, Khan, Ekhzaimy, Masood, \& Sakharkar, 2016)

El presente desarrollo investigativo se centra en plasmar la utilidad de la Hemoglobina Glicosilada (HbA1c) como una prueba versátil con diferentes usos en los pacientes que presentan diabetes tipo 2, a los fines de asistir al personal de salud en cuanto a las ventajas y desventajas de la prueba, sus diferentes usos y las actualizaciones de las principales guías clínicas de la diabetes tipo 2, como lo son las emitidas por la Asociación Latinoamericana de Diabetes (ALAD) y la Asociación Americana de la Diabetes (ADA). Asimismo, se pretende aportar a cualquier tipo de lector un material bibliográfico de valor cientificoacadémico que sirva de base para el fortalecimiento de nuevos conocimientos, especialmente para pacientes diabéticos cuyo conocimiento es un elemento importante para participar en el manejo de su enfermedad y en la mejora de su pronóstico.

\section{Materiales y Métodos}

La presente investigación tiene como objetivo general una revisión documental de 
material bibliográfico encontrado completa y libremente por medio de algunas bases de datos, entre las que se encuentran: Intramed, Mediagraphic, SciELO, NCBI, ELSEIVER, entre otras; así como también en otros portales web de importantes organizaciones como la OMS, OPS, MedlinePlus, Asociación Latinoamericana de Diabetes (ALAD), Instituto Nacional de la Diabetes y las Enfermedades Digestivas y Renales de los Estados Unidos, entre otras; en virtud de lo cual, disponer de computadoras personales con conexión a internet, resultó un indispensable recurso material.

La exploración, efectuada, se basó en el uso de los siguientes descriptores de búsqueda: "Hemoglobina Glucosilada", "Diabetes tipo 2" y "Hemoglobina Glicosilada en Diabetes tipo 2"; la información encontrada se filtró bajo los criterios de idioma del contenido (español e inglés) y rango de fecha de publicación (los últimos ocho años), en definitiva, representaron las otras variables que en mayor medida definieron una primera fase de investigación.

Esta investigación documental, efectuada en el marco de una metodología de revisión, estuvo enfocada en recabar información sobre la utilidad de la prueba de Hemoglobina Glicosilada en pacientes con diabetes tipo 2 y para ello, el material bibliográfico que se consideró rescatar, estuvo representado por artículos originales y de revisión, publicados en revistas científicas. De la misma manera se consideró otros tipos de contenidos, preferiblemente producidos o promovidos por instituciones, entes, organizaciones, sociedades o asociaciones de carácter público o privado; nacionales, internacionales o multilaterales; de procedencia Americana o Europea; inclusive de cualquier otra región de habla hispana o anglosajona que facilitaran de manera completa y gratuita el acceso a cualquier otro recurso en formato electrónico o digital, archivos o contenidos definidos como protocolos, consensos, manuales, guías clínicas, boletines informativos, informes, entre otros.
Seguidamente, como segunda y última fase del proceso investigativo, se compartió y distribuyó el total del material bibliográfico seleccionado entre los integrantes del equipo, a los fines de proceder con la lectura crítica de cada uno de ellos, y en definitiva se llevó a cabo una discusión grupal para determinar los consensos necesarios que llevaron al desarrollo de los resultados y conclusiones.

\section{Resultados}

Tradicionalmente el diagnóstico de diabetes se ha realizado por medio de la determinación de glucemia en diferentes circunstancias: ayunas, al azar o durante una prueba de tolerancia oral a la glucosa (PTG). Otro instrumento para su diagnóstico es la hemoglobina glucosilada (HbA1c). González et al. (2015) Realizaron un estudio transversal en 200 pacientes no diabéticos atendidos en la sala de exámenes preventivos del Hospital Militar Central "Dr. Carlos J. Finlay", entre julio de 2012 y junio de 2013, con la finalidad de evaluar el uso de la $\mathrm{HbA} 1 \mathrm{c}$ en el diagnóstico precoz de la diabetes mellitus en relación con la glucemia en ayunas. Los autores concluyeron: "que la $\mathrm{HbA} 1 \mathrm{c}$ con valor de corte $\geq 6,5 \%$ es una herramienta útil para confirmar el diagnóstico de diabetes mellitus, sin embargo, la glucemia en ayunas es más adecuada para estudios de pesquisa". (p. 52, 59)

La prueba de hemoglobina glicosilada o glucosilada $(\mathrm{HbA1c})$ es un examen de sangre para la diabetes tipo 2 y prediabetes. Esta prueba mide el nivel promedio de glucosa o azúcar en la sangre durante los últimos tres meses. La prueba puede usarse sola o en combinación con otras pruebas de diabetes para hacer un diagnóstico. Asimismo, la $\mathrm{HbA} 1 \mathrm{c}$ se usa con la finalidad de controlar el manejo de la diabetes. "Esta prueba es diferente a los controles de azúcar en la sangre que las personas con diabetes se hacen todos los días." (Enciclopedia Médica ADAM, 2019) 
Existen varias ventajas conocidas de la HbA1c sobre la glucemia en ayunas y la PTG, entre las cuales tenemos: "mayor conveniencia debido a que no es necesario el estado de ayunas, mayor estabilidad preanalítica y menores variaciones de un día a otro por causa de estrés o enfermedad". (González, y otros, 2015, p. 52)

En contraparte, Pereira, Palay, Rodríguez, Neyra, \& Chía (2015) reconocen las siguientes limitaciones de la HbA1c como método diagnóstico:

- Mayor costo

- Disponibilidad limitada

- Correlación incompleta entre A1c y promedio de glucemias en algunos individuos

- Resultado desorientador en pacientes con hemoglobinopatías (Hb S, C, F Y E) y ciertas anemias (hemolíticas, ferropénicas)

- No es válido en mujeres embarazadas ni para el diagnóstico de DM de tipo 1. (Ante todas estas situaciones se debe continuar con glucemias en ayuna y $2 \mathrm{~h}$ PG). (p. 557)

La HbA1c entre sus usos, puede ayudar a los profesionales de la salud a:

- Detectar la prediabetes en una persona y aconsejarla sobre cambios en el estilo de vida para ayudar a retrasar o prevenir la diabetes tipo 2.

- Detectar la diabetes tipo 2.

- Trabajar con la persona para vigilar la enfermedad y ayudar a tomar decisiones de tratamiento para prevenir complicaciones. (Instituto Nacional de la Diabetes y las Enfermedades Digestivas y Renales de los Estados Unidos, 2019)

La HbA1c proporciona una medida fiable de la glucemia crónica y se correlaciona bien con el riesgo de complicaciones de la diabetes a largo plazo, por lo que actualmente se considera la prueba de elección para el seguimiento y el tratamiento crónico de la diabetes. Sin embargo, el punto de corte de la $\mathrm{HbA} 1 \mathrm{c}$ desde el punto de vista diagnóstico sigue siendo controvertido. Entre los diabéticos, los niveles de glucosa en sangre aumentan y la glucosa se adhiere a la molécula de hemoglobina de manera dependiente de la concentración. La hemoglobina unida a glucosa (glicada) o HbA1c proporciona los niveles medios de glucosa en la sangre de un individuo a medida que se glica con la hemoglobina. Es importante señalar que los niveles de $\mathrm{HbA} 1 \mathrm{c}$ son directamente proporcionales a los niveles de glucosa en sangre. Una prueba simple de glucosa en sangre, como una prueba de glucosa en ayunas (FGT), es una medida de la concentración de glucosa presente en la sangre de un individuo en un momento determinado. (Sherwani, Khan, Ekhzaimy, Masood, \& Sakharkar, 2016)

Pardo, García, Gallego, \& Cachón (2019) al respecto de la hemoglobina glucosilada (HbA1c) refieren lo siguiente:

Es el principal parámetro recomendado para documentar el control glucémico de los últimos 3 meses en pacientes diabéticos; se reconoce como indicador intermedio de proceso y de resultados. Su buen control ha demostrado ser un punto de referencia para la mejora de la mayoría de las complicaciones por diabetes y demuestra ser útil para la prevención y retraso de las posibles complicaciones crónicas. Por tanto, uno de los principales pilares para el control del diabético tipo 2 es la medición de la HbA1c y es la principal herramienta para el manejo y ajuste de tratamiento en el paciente diabético. Se ha observado que el control de la $\mathrm{HbA1c}$ en el centro de salud incrementa la frecuencia y eficiencia de la terapia y, a su vez, disminuye los valores de HbA1c. Existen distintos métodos para la determinación de HbA1c: los más usados son los métodos realizados en laboratorio, pero también disponemos de métodos rápidos con anali-

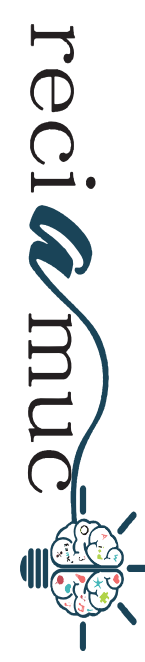


zadores que se pueden usar en la consulta y así facilitar las decisiones terapéuticas en el momento, lo que disminuye el número de consultas. (p. 147)

Asimismo, brinda potenciales pronósticos ya que la HbA1c no solo es un biomarcador útil del control glucémico a largo plazo, sino también un buen predictor del perfil lipídico; por lo que, la monitorización del control glucémico con HbA1c podría tener beneficios adicionales en la identificación de aquellos pacientes con diabetes con alto riesgo de complicaciones cardiovasculares. En tal sentido, se puede aseverar que la $\mathrm{HbA} 1 \mathrm{c}$ es la única prueba que proporciona información valiosa la cual puede usarse para el tratamiento de enfermedades crónicas. En sujetos que pueden tener diabetes, ha sido identificado un nivel elevado de $\mathrm{HbA} 1 \mathrm{c}$ como un factor de riesgo significativo de enfermedades cardiovasculares y accidente cerebrovascular. Asimismo, los niveles altos de $\mathrm{HbA1c}$ se relacionan con un mayor riesgo de recurrencia de taquiarritmia auricular en estos pacientes y fibrilación auricular paroxística sometidos a ablación con catéter. Es importante destacar que, incluso un aumento del 1\% en la concentración de $\mathrm{HbA} 1 \mathrm{c}$ se relacionó con un incremento del $30 \%$ aproximadamente, en la mortalidad por todas las causas y un incremento del $40 \%$ en la mortalidad por cardiopatía isquémica o cardiovascular entre los pacientes diabéticos. Mientras que bajar el nivel de $\mathrm{HbA} 1 \mathrm{c}$ en un $0,2 \%$ podría reducir la mortalidad en un 10\%. (Sherwani, Khan, Ekhzaimy, Masood, \& Sakharkar, 2016)

Las principales actualizaciones en las guías clínicas de la Asociación Latinoamericana de Diabates - ALAD (2019), con respecto a la $\mathrm{HbA} 1 \mathrm{c}$ son las siguientes:

- Como prueba diagnóstica no se recomienda el uso de la determinación de la Hba1c como método único que sustituya a los métodos actuales basados en la determinación de la glucemia, para el diagnóstico de los estados de disgluce- mia previos a la diabetes en la población adulta en Latinoamérica.

- Para mantener el control glicémico en pacientes con diabetes tipo 2 estables hospitalizados en una sala de medicina general por enfermedad interrecurrente, se recomienda realizar $\mathrm{HbA} 1 \mathrm{c}$ en todos los pacientes con diabetes o hiperglucemia (glucosa mayor a $140 \mathrm{mg} / \mathrm{dL}$ ) que ingresan a hospital si no se ha realizado en los últimos 3 meses.

- En la prevención de enfermedades coronarias en pacientes con diabetes tipo 2 , se ha observado que el control intensivo de la glucosa $(\mathrm{HbA} 1 \mathrm{c}<6 \%$ en comparación con 7-7.9\%), puede reducir la incidencia de infarto del miocardio, revascularización coronaria y angina inestable. A medida que las concentraciones de $\mathrm{HbA} 1 \mathrm{c}$ disminuyen, también disminuye el riesgo de eventos cardiovasculares, sin embargo, se ha observado también un incremento en la mortalidad en los sujetos en el grupo de tratamiento intensivo. Recomendamos la reducción de la $\mathrm{HbA} 1 \mathrm{c}$ aproximadamente de 1\% para una reducción del riesgo relativo de infarto del miocardio no fatal, sin beneficiar el riesgo de EVC y la mortalidad. Se sugiere que el control intensivo de la glucosa se prescriba adecuadamente de forma individualizada, tomando en cuenta la edad, la duración de la diabetes, y la historia de enfermedad cardiovascular. La ALAD recomienda el control intensivo de la glucosa ( $\mathrm{HbA} 1 \mathrm{c}<6.0 \%)$ para reducir la incidencia de infarto agudo al miocardio; sin embargo, debe prescribirse de forma individualizada, omitiendo a individuos de edad avanzada y con mayor duración de la diabetes, en especial cuando presenten complicaciones avanzadas. (Evidencia Alta).

- En casos de insuficiencia cardíaca en personas adultas con diabetes tipo 2, cada incremento de $1 \%$ en la HbA1c por arriba de las metas, y el tratamiento con 
tiazolidinedionas se asocian con mayor incidencia de insuficiencia cardiaca.

- Con relación a la Enfermedad cerebrovascular en personas adultas con diabetes tipo 2, esta guía coincide con la guía del Comité de Expertos de la Asociación Canadiense de Diabetes para EVC, en donde se recomienda que a todos los pacientes con un ataque isquémico transitorio (AIT) se les realice una glucosa de ayuno, HbA1c o pruebas de tolerancia oral con 75 gr de glucosa para descartar diabetes poco tiempo después de ser egresados del hospital. Todos los pacientes con diabetes y EVC o AIT deben recibir el mismo tratamiento que se recomienda para los pacientes sin diabetes. (Evidencia Alta). (p. 13 - 98)

Dentro de la actualización anual de su documento "Estándares de atención médica en la diabetes" la Asociación Americana de la Diabetes (2019) refiere en cuanto a los objetivos glucémicos para la HbA1C, las siguientes recomendaciones:

- Un objetivo A1C razonable para muchos adultos no embarazados es $<7 \%$ (53 $\mathrm{mmol} / \mathrm{mol}$ ).

- Los proveedores podrían sugerir razonablemente objetivos $\mathrm{A} 1 \mathrm{C}$ más estrictos (como <6.5\% [48 mmol / mol]) para pacientes individuales seleccionados si esto se puede lograr sin hipoglucemia significativa $u$ otros efectos adversos del tratamiento (es decir, polifarmacia). Los pacientes apropiados podrían incluir a aquellos con diabetes de corta duración, diabetes tipo 2 tratada solo con estilo de vida o metformina, larga vida útil o sin enfermedad cardiovascular significativa.

- Los objetivos A1C menos estrictos (como $<8 \%$ [64 mmol / mol]) pueden ser apropiados para pacientes con antecedentes de hipoglucemia severa, esperanza de vida limitada, complicaciones microvasculares o macrovasculares avanza- das, condiciones comórbidas extensas o diabetes de larga duración en quienes el objetivo es difícil de lograr a pesar de la educación para el autocontrol de la diabetes, el control adecuado de la glucosa y las dosis efectivas de múltiples agentes reductores de la glucosa, incluida la insulina. (p. S5)

\section{Conclusión}

Después de haber realizado esta revisión se concluye que, la HbA1c constituye en la actualidad la prueba de elección para el seguimiento y el tratamiento crónico de la diabetes, especialmente de la diabetes tipo 2 , no obstante, desde el punto de vista diagnóstico sigue siendo un tanto controvertido.

La versatilidad de sus usos la convierten en una gran herramienta en la prevención, diagnóstico, tratamiento, control y pronósticos de complicaciones del paciente con diabetes tipo 2. Algunos autores, así como la ALAD consideran que la HbA1c como prueba diagnóstica para los estados de disglucemia y diabetes, debe usarse en combinación con otras pruebas de diabetes como la glucemia en ayunas y la PTG.

En el control del diabético tipo 2 la medición de la HbA1c es la principal herramienta que permite el adecuado manejo y ajuste de tratamiento. Asimismo, resulta un predictor importante de complicaciones asociadas a esta enfermedad. En virtud de lo cual, resulta fundamental trabajar con el paciente para vigilar la enfermedad por medio de esta prueba y ayudar a tomar decisiones de tratamiento para prevenir cualquiera de sus complicaciones.

\section{Bibliografía}

Asociación Americana de la Diabetes. (Enero de 2019). Resumen de revisiones: Estándares de atención médica en diabetes 2019. Diabetes Care, 42(1), S4-S6. doi:https://doi.org/10.2337/ dc19-Srev01

Asociación Latinoamericana de Diabates - ALAD. (2019). Guías ALAD sobre el Diagnóstico, Control y Tratamiento de la Diabetes Mellitus Tipo 2

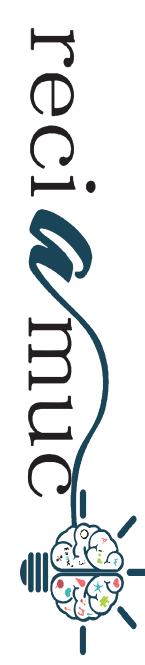




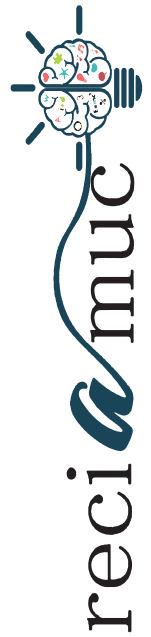

con Medicina Basada en Evidencia Edición 2019. Asociación Latinoamericana de Diabates - ALAD. Ciudad de México: PERMANYER. Recuperado el 15 de Julio de 2020, de http://revistaalad.com/ guias/5600AX191_guias_alad_2019.pdf

Enciclopedia Médica ADAM. (24 de Mayo de 2019). MedlinePlus. Recuperado el 03 de Julio de 2020, de https://medlineplus.gov/spanish/a1c.html\#: :text=La\%20prueba\%20de\%20hemoglobina\%20 glicosilada, diabetes $\% 20$ para $\% 20$ hacer $\% 20$ un\%20diagn\%C3\%B3stico.

González, R., Aldama, I., Fernández, L., Ponte, I., Rivero, M., \& Castillo, N. (2015). Hemoglobina glucosilada para el diagnóstico dediabetes mellitus en exámenes médicos preventivos. Revista Cubana de Medicina Militar, 44(1), 50-62. Recuperado el 08 de Julio de 2020, de http://scielo.sld.cu/pdf/mil/ v44n1/mil07115.pdf

Instituto Nacional de la Diabetes y las Enfermedades Digestivas y Renales de los Estados Unidos. (Abril de 2019). Institutos Nacionales de la Salud de los Estados Unidos. Instituto Nacional de la Diabetes y las Enfermedades Digestivas y Renales de los Estados Unidos. Recuperado el 05 de julio de 2020, de https://www.niddk.nih.gov/health-information/ informacion-de-la-salud/diabetes/informacion-general/pruebas-diagnostico/prueba-a1c-diabetes

Mayo Clinic. (16 de Marzo de 2019). Mayo Clinic. Recuperado el 07 de Julio de 2020, de https:// www.mayoclinic.org/es-es/diseases-conditions/ type-2-diabetes/symptoms-causes/syc-20351193

OMS. (08 de Junio de 2020). Organización Mundial de la Salud - OMS. Recuperado el 03 de Julio de 2020, de https://www.who.int/es/news-room/factsheets/detail/diabetes

OPS. (2012). Organización Panamericana de la Salud - OPS. Recuperado el 07 de Julio de 2020, de https://www.paho.org/hq/index.php?option=com_
content\&view=article\&id=6715:2012-diabetes\&Itemid=39446\&lang=es

OPS. (2019). Organización Panamericana de la Salud - OPS. Recuperado el 05 de Julio de 2020, de https://www.paho.org/hq/index.php?option=com_content\&view=article\&id=14780: world-diabetes-day-2018-diabetes-concerns-every-family\&ltemid=1969\&lang=es

Organización Mundial de la Salud - OMS. (2016). Organización Mundial de la Salud. Recuperado el 04 de julio de 2020, de https://apps.who.int/ iris/bitstream/handle/10665/204877/WHO_NMH NVI_16.3_spa.pdf; jsessionid $=80 \mathrm{DB} 8600 \mathrm{E} 7 \mathrm{~B} 6 \mathrm{~F}-$ 0C8B8068F6D7754E2CE? sequence $=1$

Organización Panamericana de la Salud - OPS. (2017). Organización Panamericana de la Salud - OPS. Recuperado el 06 de Julio de 2020, de https://www.paho.org/hq/index.php?option=com_ content\&view $=$ category \&id $=4475 \&$ layout $=$ blog $\&$ Itemid $=40610 \&$ lang $=$ es \& limitstart $=15$

Pardo, G., García, P., Gallego, M. Á., \& Cachón, M. L. (2019). Control del diabético tipo 2 en atención primaria utilizando un dispositivo de medición capilar de hemoglobina glucosilada. Medicina General y de Familia, 8(4), 146-150. Recuperado el 20 de Julio de 2020, de http://mgyf.org/wp-content/ uploads/2019/11/MGYF2019_044.pdf

Pereira, O. L., Palay, M., Rodríguez, A., Neyra, R., \& Chía, M. d. (2015). Hemoglobina Glucosilada en Pacientes con Daibetes Mellitus. MEDISAN, 19(4), 555-561. Recuperado el 08 de Julio de 2020, de http://scielo.sld.cu/pdf/san/v19n4/san12194.pdf

Sherwani, S. I., Khan, H. A., Ekhzaimy, A., Masood, A., \& Sakharkar, M. K. (2016). Importancia de la prueba de HbA1c en el diagnóstico y pronóstico de pacientes diabéticos. Perspectivas de Biomark, 11, 95-104. doi:10.4137 / BMI.S38440

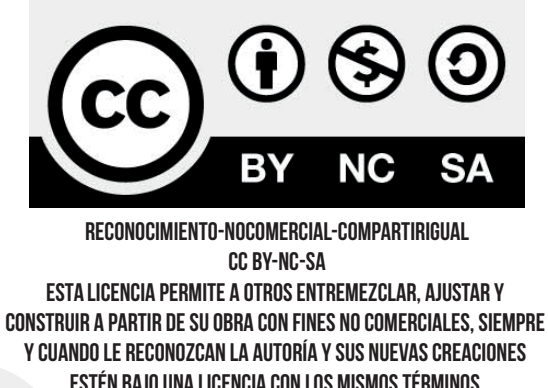

\section{CITAR ESTE ARTICULO:}

Flores Poveda, K., Quiñonez García, K., Flores Subía, D., \& Cárdenas Choez, C. (2020). Utilidad de hemoglobina glicosilada en diabetes tipo 2. RECIAMUC, 4(3), 118-126. doi:10.26820/reciamuc/4.(3).julio.2020.118-126 